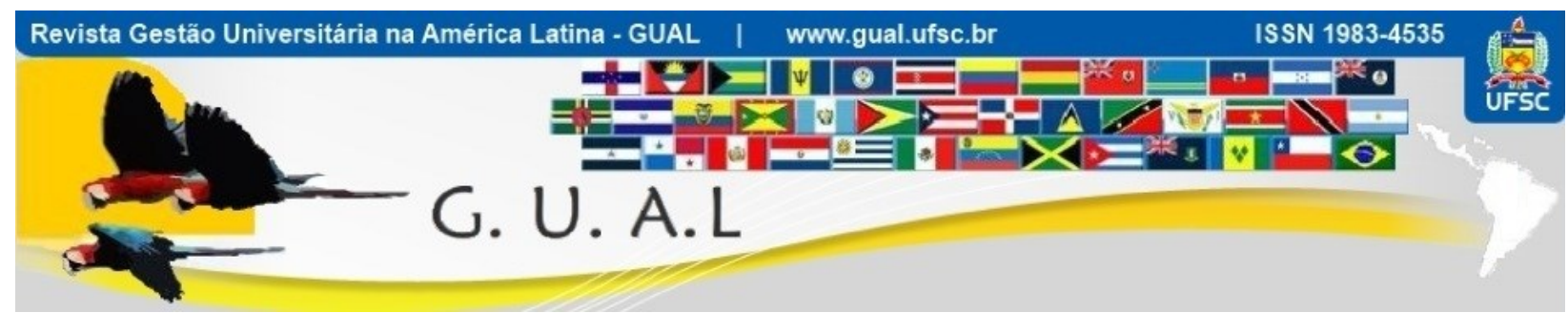

DOI: https://doi.org/10.5007/1983-4535.2021.e74645

\title{
REDES INTERINSTITUCIONAIS NA PERSPECTIVA DA HÉLICE TRÍPLICE: O CASO DE UMA UNIVERSIDADE PÚBLICA DO SUL DO BRASIL
}

\author{
INTERINSTITUTIONAL NETWORKS IN THE PERSPECTIVE OF THE TRIPLE \\ HELIX: A CASE OF A SOUTH BRAZIL PUBLIC UNIVERSITY
}

\author{
Silvana Silva Vieira Tambosi, Doutora \\ http://orcid.org/0000-0002-4762-7957 \\ profa.silvana.vieira@gmail.com \\ Universidade Regional de Blumenau | Programa de Pós-graduação em Ciências Contábeis \\ Blumenau | Santa Catarina | Brasil \\ Maria Jose Carvalho de Souza Domingues, Doutora \\ http://orcid.org/0000-0001-7771-144X \\ mariadomingues@furb.br \\ Universidade Regional de Blumenau | Programa de Pós-graduação em Ciências Contábeis \\ Blumenau | Santa Catarina | Brasil \\ Iara Regina dos Santos Parisotto, Doutora \\ http://orcid.org/0000-0003-1859-6394 \\ iaraparisotto@furb.br \\ Universidade Regional de Blumenau | Programa de Pós-graduação em Ciências Contábeis \\ Blumenau | Santa Catarina | Brasil
}

Recebido em 10/junho/2020

Aprovado em 09/novembro/2020

Publicado em 01/janeiro/2021

Sistema de Avaliação: Double Blind Review

Agência de fomento: CAPES - Coordenação de Aperfeiçoamento de Pessoal de Nível Superior

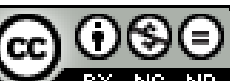

Esta obra está sob uma Licença Creative Commons Atribuição-Uso. 


\title{
RESUMO
}

O objetivo dessa pesquisa consistiu em analisar a percepção dos atores universitários acerca das parcerias interinstitucionais, na perspectiva de uma universidade pública municipal, como membro da hélice tríplice. Para isto, adotou-se, em um primeiro momento a abordagem qualitativa, que consistiu em mapear as redes de parcerias interinstitucionalizadas por meio de convênios entre empresas, governo e universidade (hélice tríplice). Em seguida, utilizou-se uma abordagem quantitativa com base na pesquisa de Rodrigues; Esther e Andrade (2017), com o uso da análise descritiva, a técnica multivariada (alfa de cronbach, cálculo de média e desvio padrão das dimensões), e a multicriterial por meio da técnica de entropia da informação. Os principais resultados demonstraram que na percepção dos atores universitários é papel da universidade oferecer ensino, pesquisa e extensão, produzir e difundir conhecimento; há ausência de clareza entre os respondentes se a relação entre a universidade com o ambiente externo pode trazer riscos para a universidade. Além disso, verificou-se que as dimensões que obtiveram as maiores pontuações da escala, foram: "os motivos que leva a universidade a se aproximar de empresas e governos" (Dim. 3), e "o propósito da universidade buscar parcerias, sob a perspectiva da dependência de recursos" (Dim. 4).

Palavras-chave: Universidade. Redes Interinstitucionais. Hélice Tríplice.

\begin{abstract}
This research aimed to analyze university actors' perception of inter-institutional partnerships, from a municipal public university's perspective, as a triple helix member. The qualitative approach was initially adopted, which consisted of mapping the inter-institutional partnership networks through agreements between companies, government, and University (triple helix). A quantitative approach based on Rodrigues's research was used; Esther and Andrade (2017) used descriptive analysis, multivariate technique (Cronbach's alpha, calculation of mean and standard deviation of dimensions) multicriterial utilizing the method of information entropy. The main results showed that in the perception of university actors, it is the University's role to offer teaching, research, and extension, to produce and disseminate knowledge; There is a lack of clarity among respondents as to whether the relationship between the University and the external environment may pose risks to the University. Moreover, it was found that the dimensions that obtained the highest scores on the scale were: "the reasons that lead the university to approach companies and governments" (Dim. 3), and "the purpose of the university to seek partnerships, under the perspective of resource dependence" (Dim. 4).
\end{abstract}

Keywords: University. Interinstitutional Partnerships. Triple Helix. 


\section{REDES INTERINSTITUCIONAIS NA PERSPECTIVA DA HÉLICE TRÍPLICE: O CASO DE UMA \\ UNIVERSIDADE PÚBLICA DO SUL DO BRASIL \\ DOI: https://doi.org/10.5007/1983-4535.2021.e74645}

\section{INTRODUÇÃO}

As universidades passam por um momento de reengenharia em seus processos, a fim de atender as novas demandas do mercado, da sociedade e dos seus estudantes. Desta forma, os estudos na área de gestão universitária vêm ganhando protagonismo no campo da administração. De acordo com Schilickmann e Melo (2012) a reengenharia é necessária nas universidades, em especial, nas universidades públicas, pois apresentam receitas que não advém da sua produtividade.

Ainda, segundo os autores há um distanciamento dos cursos oferecidos e as demandas do mercado, no que tange às necessidades da sociedade. Os estudantes, não se formam com as habilidades exigidas de sua futura área de atuação. Neste sentindo, discute-se a utilidade da universidade, que por sua vez, deve proporcionar uma formação voltada para o mercado de trabalho (SCHILICKMANN; MELO, 2012). Desta forma, o desafio é repensar a estrutura da universidade, derivando em mudança internas e novos modos de parecerias com o ambiente externo. (COLOMBO, 2013; MEYER Jr, 2014). Esta é uma alternativa para que as universidades possam responder melhor as pressões do mercado, atuando em um sistema interligado.

A constituição de redes de cooperação entre a universidade, as empresas e os órgãos de fomento, com o intuito de viabilização de projetos, oportunizam uma constante aproximação e atualização da academia com o mercado. Nesta relação todos os envolvidos possuem ganhos. Frequentemente, as empresas visualizam nas universidades uma oportunidade de atender o seu esforço tecnológico, como fonte de vantagem competitiva, uma vez que há o abrandamento de custos com o desenvolvimento de pesquisas (PORTO et al., 2011).

Os autores Melo (2002); Rodrigues; Esther e Andrade (2017) salientam que, a universidade em virtude da sua contribuição para a produção de conhecimento, tem em si um potencial para a inovação. Nesta perspectiva, segundo Etzkowitz (1994); Etzkowitz e Leydesdorff (2000); Etzkowitz; Zhou e Chunyan (2017) a hélice tríplice é um modelo composto por instituições de ensino, empresas e governo para o fomento da inovação e do desenvolvimento econômico. De acordo com Pfeffer e Salancik (1978); Ulrich e Barney (1984); Balestrin; Verschoore e Reyes (2010) as organizações, ao formar parcerias, tem o propósito de compartilhar e ter acesso a recursos que são escassos. 


\section{REDES INTERINSTITUCIONAIS NA PERSPECTIVA DA HÉLICE TRÍPLICE: O CASO DE UMA \\ UNIVERSIDADE PÚBLICA DO SUL DO BRASIL \\ DOI: https://doi.org/10.5007/1983-4535.2021.e74645}

Sob esta perspectiva, levantou-se a seguinte questão de pesquisa: qual a percepção dos atores universitários acerca das parcerias interinstitucionais, na perspectiva de uma universidade pública municipal, como membro da hélice tríplice? As universidades possuem um relevante papel na sociedade, desta maneira, busca-se reaver se há intercorrência na percepção dos atores universitários acerca desta temática.

\section{REDES INTERINSTITUCIONAIS E HÉLICE TRÍPLICE NA GESTÃO ESTRATÉGICA EM INSTITUIÇÕES DE ENSINO SUPERIOR}

A gestão educacional, como um campo de estudo e prática, foi provinda dos princípios de gestão, utilizados inicialmente nos ramos da indústria e do comércio, principalmente nos Estados Unidos. Desta forma, o seu desenvolvimento teórico, ocorreu a partir da aplicação de modelos industriais aplicados em contextos educacionais. Ao passo que tornou-se um campo acadêmico, os teóricos e gestores da área começaram a desenvolver modelos alternativos, com base em suas observações e experiências em instituições de ensino (BUSH, 2006).

Faz-se necessário uma reengenharia nas universidades. Em especial, as universidades públicas que apresentam receitas que não advém da sua produtividade. Há um enorme distanciamento dos cursos oferecidos e as demandas do mercado, no que tange às necessidades da sociedade. Os acadêmicos, em geral, não se formam com as habilidades exigidas de sua futura área de atuação. Discute-se a "utilidade da universidade", que por sua vez, deve proporcionar um serviço que seja útil para os estudantes que tem por objetivo uma formação voltada para o mercado de trabalho (SCHILICKMANN; MELO, 2012).

As universidades possuem um relevante papel na sociedade, em vista disto, o desempenho dessas instituições carece de maior atenção de pesquisadores e gestores da área da administração universitária. Pontos decorrentes da complexidade organizacional, que envolve uma universidade, que muitas vezes possui ambiguidade de objetivos; a estrutura decisória ocorre de forma colegiada; há uma pluralidade de interesses; a essência do processo educacional, principalmente na transferência do conhecimento e o aprendizado, assim como a promoção de valores humanos, possui recursos escassos e demandam de uma administração apropriada e de uma teoria própria (MEYER Jr, 2014).

No cenário de competição atual, em que as instituições de ensino superior estão inseridas, são demandadas a buscar novas formas de atuação e de melhoria da qualidade dos serviços educacionais, a fim de obter desempenho e valor dos serviços oferecidos. Para tal, é necessária uma gestão com liderança e determinação, para atuarem em estruturas mais 


\section{REDES INTERINSTITUCIONAIS NA PERSPECTIVA DA HÉLICE TRÍPLICE: O CASO DE UMA \\ UNIVERSIDADE PÚBLICA DO SUL DO BRASIL \\ DOI: https://doi.org/10.5007/1983-4535.2021.e74645}

flexíveis direcionadas aos consumidores e aos processos. Assim, o desafio é repensar sua estrutura, decorrendo em mudança internas e novos modos de engajamento externo. (COLOMBO, 2013; MEYER Jr, 2014).

Uma alternativa é a possibilidade de as instituições de ensino responderem melhor as demandas do mercado, atuando em um sistema interligado. O conceito de instituições com este entendimento, pode apresentar um efeito vultoso sobre as contingencias existentes acerca das organizações. O autor Weick (1976) na década de 1970 já indicava o desenvolvimento de instrumentos conceituais, que preservassem os sistemas interligados, tais como: discorrer sobre os elementos existentes em instituições de ensino que possam ser integrados; a elaboração de uma metodologia contextual; a coleta e descrição das estruturas para um sistema interligado; a especificação da tecnologia fundamental para as instituições educacionais (WEICK, 1976).

Neste sentido, a formação de redes de cooperação entre universidade, empresas e órgãos de fomento, para a viabilização de projetos, contribuem para a aproximação e constante atualização da comunidade acadêmica com o mercado. Nesta relação, todos os envolvidos possuem ganhos. "As motivações que levam à cooperação são distintas para as universidades e as empresas, bem como suas características e necessidades, a intersecção destas encontra-se na necessidade de inovar.” (PORTO et al., 2011, p. 53).

Segundo Porto (2006) as universidades são tidas como um receptáculo de conhecimento, cujo o acesso ocorre por meio de parcerias, licenciamentos, qualificação dos seus atores e desenvolvimento de pesquisas. Comumente, as organizações veem nas universidades um meio de suplementar o seu esforço tecnológico, como fonte de vantagem competitiva com a atenuação de custos com pesquisas. Pois, as organizações possuem recursos internos restritos (profissionais qualificados e recursos financeiros) para a produção de pesquisa.

As universidades veem buscando atender as necessidades do mercado, pelo senso de utilidade da universidade. Isto reforça a tendência de sua reengenharia em seus modelos e processos organizacionais, conforme destacado por Schilickmann e Melo (2012). Cabe destacar que, devido a sua contribuição para a produção de conhecimento, a Universidade possui potencial para a inovação (MELO, 2002; RODRIGUES; ESTHER; ANDRADE, 2017). 


\section{REDES INTERINSTITUCIONAIS NA PERSPECTIVA DA HÉLICE TRÍPLICE: O CASO DE UMA \\ UNIVERSIDADE PÚBLICA DO SUL DO BRASIL \\ DOI: https://doi.org/10.5007/1983-4535.2021.e74645}

Nesta perspectiva, a hélice tríplice é um modelo que integra instituições de ensino, empresas e governo para o fomento da inovação e desenvolvimento econômico (ETZKOWITZ, 1994; ETZKOWITZ; LEYDESDORFF, 2000; ETZKOWITZ; ZHOU; CHUNYAN, 2017). O termo foi originado metaforicamente, o intuito era a identificação dos envolvidos de um sistema de inovação (âmbito regional), de uma determinada rota nos Estados Unidos da América. Atualmente, o termo hélice tríplice é reconhecido internacionalmente nos estudos, na orientação de práticas e políticas de inovação em âmbitos local até multinacional. O objetivo é aprimorar as “interações universidade-indústriagoverno.” (ETZKOWITZ; ZHOU, 2017, p.23).

Este modelo constitui um meio para melhorar as condições de inovação em uma sociedade baseada no conhecimento. Neste arranjo, a indústria é tida como o local da produção; o governo como fonte de relações contratuais que assegurara a estabilidade das interações; e a universidade produz novos conhecimentos e tecnologia. Estas inter-relações constituem o princípio gerador das economias baseadas no conhecimento (ETZKOWITZ, 2003).

Ainda, segundo Etzkowitz e Zhou (2017, p. 23) na perspectiva da hélice tríplice "a universidade está deixando de ter um papel social secundário, ainda que importante, de prover ensino superior e pesquisa, e está assumindo um papel primordial equivalente ao da indústria e do governo, como geradora de novas indústrias e empresas."

Etzkowitz (1994), elucida que a hélice tríplice possibilita o levantamento de recursos externos à universidade, além do governo, pois pode-se contar com a iniciativa privada para a fonte de financiamento de projetos. Com base nesta premissa, o estudo de Rodrigues; Esther e Andrade (2017, p.02) buscou "compreender qual efetivamente está sendo o caminho trilhado na estruturação desta relação, levantando, para isto, a situação específica de uma universidade brasileira." Os resultados demonstraram uma ausência consensual acerca da postura e visão dos atores institucionais acerca da relação da universidade com o ambiente externo.

Apesar de não ter havido um consenso nos resultados da pesquisa de Rodrigues; Esther e Andrade (2017) quanto a relação da universidade com o ambiente externo, os autores reconhecem a importância da relação entre a universidade, governo e empresa, atuando em rede de cooperação, para a obtenção de recursos, uma vez que existe uma dependência de recursos destas instituições. 


\section{REDES INTERINSTITUCIONAIS NA PERSPECTIVA DA HÉLICE TRÍPLICE: O CASO DE UMA \\ UNIVERSIDADE PÚBLICA DO SUL DO BRASIL \\ DOI: https://doi.org/10.5007/1983-4535.2021.e74645}

Com relação a dependência de recursos, trata-se de uma abordagem utilizada nos estudos sobre redes de cooperação. O ponto de interesse é compreender o processo pelo qual as organizações diminuem suas dependências do ambiente, fazendo uso de diversas estratégias a fim de ampliar seu poder. Esta teoria pormenoriza entre os tipos de dependência de recursos, sejam eles materiais ou imateriais, como potenciais determinantes na formação de redes.

Nesse sentido, destaca-se o artigo seminal de Pfeffer e Salancik (1978); Ulrich e Barney (1984); Balestrin; Verschoore e Reyes (2010) que fazem uma revisão de algumas das principais configurações de redes de cooperação e redes sociais. Na perspectiva da teoria da dependência de recursos, as organizações estruturam-se em redes, com o objetivo de compartilhar e ter acesso a recursos que são escassos.

A teoria da dependência de recursos, segundo Penrose (1959); Teece (1982); Prahalad e Bettis (1986); Barney (1991); Clegg et al. (1999) tem como unidade fundamental de análise os recursos e capacidades controlados pela empresa. Isto inclui todos os atributos que capacitam a empresa para a definição e implementação de estratégias.

Os recursos podem ser divididos em quatro tipos: financeiros, físicos, humanos e organizacionais (BARNEY, 1991; CLEGG et al., 1999). A perspectiva da dependência de recursos apoia-se em dois pressupostos referentes aos recursos e as capacidades da empresa. O primeiro pressuposto é que os recursos e as capacidades podem apresentar variações significativas entre as empresas. O segundo pressuposto assume que estas diferenças podem apresentar estabilidade, considerando a imobilidade de recursos (CLEGG et al.,1999).

\section{MÉTODOS E TÉCNICAS DE PESQUISA}

Nesta seção descreve-se o enquadramento metodológico da pesquisa e os procedimentos para coleta e análise dos dados.

\subsection{ENQUADRAMENTO METODOLÓGICO}

Com o objetivo de analisar a percepção dos atores universitários acerca das parcerias interinstitucionais, na perspectiva de uma universidade pública municipal, como membro da hélice tríplice, adotou-se, em um primeiro momento a abordagem qualitativa, que consistiu em mapear as redes de parcerias interinstitucionalizadas por meio de convênios entre empresas, governo e universidade (hélice tríplice). 


\section{REDES INTERINSTITUCIONAIS NA PERSPECTIVA DA HÉLICE TRÍPLICE: O CASO DE UMA \\ UNIVERSIDADE PÚBLICA DO SUL DO BRASIL \\ DOI: https://doi.org/10.5007/1983-4535.2021.e74645}

Para o mapeamento dos projetos financiados por estas instituições parceiras e a universidade, foi necessário entrar em contato com o escritório de projetos da universidade, que estabelece os contratos de cooperação e gerenciam os recursos investidos. Em seguida, contatou-se o NIT (Núcleo de Inovação Tecnológica) da universidade, a fim de levantar e entender como ocorre a captação e a viabilização contratual com as empresas privadas. E, por último, procurou-se a Divisão de Pós-Graduação, para que fosse possível realizar um levantamento dos recursos de pesquisas (bolsas de estudos, de extensão), bem como a sua distribuição aos programas de pós-graduação.

Desta forma, foi possível constituir a base de dados. Esta foi composta por 119 professores/pesquisadores vinculados a programas de Pós-graduação (Stricto Sensu), o reitor, três pró-reitores (administração, pesquisa e extensão, de ensino), três gestores das unidades administrativas diretamente ligada as parcerias interinstitucionalizadas. Totalizando 126 participantes. Depois do mapeamento das parcerias interinstitucionalizadas e dos atores universitários envolvidos, foi necessário a utilização de uma abordagem quantitativa, tanto na abordagem quanto no tratamento dos dados, por meio de técnicas estatística descritiva, multivariada e multicritério.

A abordagem quantitativa objetiva a mensuração de fenômenos, compreendendo a coleta e a análise de dados numéricos (quantitativos), e realizando testes estatísticos. O fator relevante para a validação de estudos quantitativos é facultado por meio da confiabilidade dos indicadores estatísticos (COLLIS; HUSSEY, 2013). A técnica de pesquisa descritiva frequentemente caracteriza alguma situação contextual, sendo esta idealizada, principalmente, para mensurar as características da ocorrência de casos de pesquisa (HAIR JR et. al., 2009).

O método multicritério é uma análise sistemática que busca identificar, caracterizar e classificar os principais fatores constituintes, por meio da comparação entre as variáveis propostas em um conjunto de critérios. O intuito é oferecer possíveis alternativas sobre as preferências do grupo envolvido no processo decisório (MATZENAUER, 2003; GOMES; ARAYA; CARIGNANO, 2004; STEWART, 2011; ALMEIDA, 2013).

Nesta segunda parte, foi adotado um questionário adaptado, a partir da pesquisa de Rodrigues, Esther e Andrade (2017), que originalmente, possuiu uma abordagem qualitativa e o instrumento consistiu em entrevistas com atores universitários. Os dados levantados, foram categorizados a partir da técnica de contagem de frequência, e as variáveis mais citadas pelos entrevistados foram organizados em três dimensões, descritas no Quadro 1. Para este artigo, 


\section{REDES INTERINSTITUCIONAIS NA PERSPECTIVA DA HÉLICE TRÍPLICE: O CASO DE UMA UNIVERSIDADE PÚBLICA DO SUL DO BRASIL \\ DOI: https://doi.org/10.5007/1983-4535.2021.e74645}

adotou-se as três dimensões da pesquisa de Rodrigues, Esther e Andrade (2017), e criou-se uma quarta dimensão, nomeada de: Parcerias - A Perspectiva da Dependência De Recursos.

Quadro 1 Relação entre as questões de investigação e os itens do questionário

\begin{tabular}{|c|c|c|}
\hline Dimensões & Questão de investigação & Itens do questionário \\
\hline $\begin{array}{l}\text { D1 - Os papeis da } \\
\text { Universidade. }\end{array}$ & $\begin{array}{l}\text { Os papeis que a } \\
\text { universidade assume } \\
\text { atualmente são: }\end{array}$ & $\begin{array}{l}\text { 1. A tríade: Ensino, Pesquisa e Extensão. } \\
\text { 2.Além da tríade, também inovação e visualização de demandas } \\
\text { externas. } \\
\text { 3. Atender a tríade, mas que está aquém das suas possibilidades. } \\
\text { 4. É um Agente de desenvolvimento econômico e social. } \\
\text { 5. É produtora e difusora de conhecimento. } \\
\text { 6. A reflexão sobre o papel ideal e o papel real da universidade. }\end{array}$ \\
\hline $\begin{array}{l}\text { D2- Riscos: } \\
\text { Universidade e o } \\
\text { ambiente externo. }\end{array}$ & $\begin{array}{l}\text { Possíveis riscos para a } \\
\text { universidade em uma } \\
\text { relação mais próxima com } \\
\text { o ambiente externo: }\end{array}$ & $\begin{array}{l}\text { 7. Não há riscos para universidade. } \\
\text { 8. Desviar o foco de suas finalidades públicas para atender } \\
\text { demandas privadas. } \\
\text { 9. Os riscos dependem da forma como essa relação é } \\
\text { regulamentada. } \\
\text { 10. A universidade corre o risco de entrar com o ônus e as empresas } \\
\text { ficarem com o bônus. } \\
\text { 11. Priorizar somente a ligação com algum setor da sociedade, } \\
\text { como as Empresas. } \\
\text { 12. Benefícios somente para áreas com maior ligação e coerência } \\
\text { com as demandas de mercado. }\end{array}$ \\
\hline $\begin{array}{l}\text { D3 - Aproximação } \\
\text { da Universidade } \\
\text { com Empresa e } \\
\text { Governo: } \\
\text { Motivações. }\end{array}$ & $\begin{array}{l}\text { Motivos que levariam a } \\
\text { universidade a buscar a } \\
\text { aproximação com o } \\
\text { governo e as empresas: }\end{array}$ & $\begin{array}{l}\text { 13. Obtenção de financiamentos na iniciativa privada. } \\
\text { 14. Contribuir no desenvolvimento econômico e social. } \\
\text { 15. Alcance de legitimidade social se aproximando da sociedade. } \\
\text { 16. Proporcionar maior inserção dos alunos no mercado. } \\
\text { 17. Aproximação do objeto de estudo e maior aplicabilidade dos } \\
\text { conhecimentos. }\end{array}$ \\
\hline $\begin{array}{l}\text { D4 - Parcerias: a } \\
\text { Perspectiva da } \\
\text { Dependência De } \\
\text { Recursos. }\end{array}$ & $\begin{array}{l}\text { Motivos que levariam a } \\
\text { universidade a buscar } \\
\text { parcerias com o governo e } \\
\text { as empresas, sob a } \\
\text { perspectiva da } \\
\text { dependência de recursos: }\end{array}$ & $\begin{array}{l}\text { 18. Obtenção de recursos financeiros na iniciativa privada. } \\
\text { 19. Obtenção de recursos financeiros na esfera pública. } \\
\text { 20. Obtenção de recursos para a aquisição de equipamentos, } \\
\text { laboratórios, etc. } \\
\text { 21. Obtenção de recursos para a qualificação dos atores } \\
\text { universitários (qualificação: cursos, participação em congressos da } \\
\text { área, etc.). } \\
\text { 22. Obtenção de recursos para a contratação de profissionais } \\
\text { especializados (interno: locação de horas na Universidade). } \\
\text { 23. Obtenção de recursos para a contratação de profissionais } \\
\text { especializados (externa: contratação de terceiros). } \\
\text { 24. O uso dos recursos captados (iniciativa privada) é utilizado em } \\
\text { outros projetos da Universidade. } \\
\text { 25. O uso dos recursos captados (área pública) é utilizado em outros } \\
\text { projetos da Universidade. } \\
\text { 26. Os resultados dos recursos captados para um projeto constituem } \\
\text { em um ponto inicial para outros projetos da universidade. } \\
\text { 27. Os recursos captados, por meio dos convênios, refletem na } \\
\text { inovação dos processos da universidade (ensino, pesquisa e } \\
\text { extensão). } \\
\text { 28. O desempenho da Universidade é superior (manutenção da } \\
\text { vantagem competitiva) devido a obtenção destes recursos, através } \\
\text { dos convênios/parcerias com a iniciativa privada e pública. }\end{array}$ \\
\hline
\end{tabular}

Fonte: adaptado de Rodrigues; Esther e Andrade (2017) 


\section{REDES INTERINSTITUCIONAIS NA PERSPECTIVA DA HÉLICE TRÍPLICE: O CASO DE UMA \\ UNIVERSIDADE PÚBLICA DO SUL DO BRASIL \\ DOI: https://doi.org/10.5007/1983-4535.2021.e74645}

Para a mensuração das questões (Quadro 1), utilizou-se escala de likert de 7 pontos, sendo 1 atribuído a discordância total do respondente e 7 a concordância total do respondente com as afirmações que compuseram as questões. $O$ questionário estruturado foi disponibilizado via Google Docs no período de fevereiro e março de 2018. Foram convidados a participar da pesquisa todos os atores universitários identificados na primeira parte da pesquisa. No dia 28 de março de 2018 encerrou-se a pesquisa, contando com a participação de 43 respondentes.

Em seguida, os dados foram tabulados em planilha do software Microsoft Excel ${ }^{\circledR}$. As questões foram agrupadas de acordo com as dimensões propostas (Quadro 1). A base de dados da planilha do software Microsoft Excel ${ }^{\circledR}$ foi importada para o Software Estatístico SPSS ${ }^{\circledR}$ versão 22. A primeira análise efetuada foi o teste estatístico de confiabilidade (Alfa de Cronbach). Este indicador é uma medida de confiabilidade que varia de 0 a 1 , sendo os valores de 0,60 a 0,70 considerados o limite inferior de aceitabilidade (HAIR Jr. et al., 2009). Em seguida, calculou-se a média e desvio padrão das dimensões.

Posteriormente, foi realizada a análise multicriterial de entropia da informação. Este método é utilizado para determinar o peso da informação presente nos indicadores avaliados (ZELENY, 1982; ZOU; YI; SUN, 2006). Originalmente o termo entropia procedeu-se da termodinâmica, e com o tempo foi sendo utilizado em outras áreas do conhecimento, como as ciências sociais (ROCHA et al, 2011). Para o cálculo da entropia, foi necessário um ponto de referência, isto é, um valor âncora em referência ao valor em que é calculada a entropia da informação. Nesta pesquisa o valor atribuído foi 7 (Concordo Totalmente). Desta forma, o valor da entropia alcançado recomendará o quanto de informação está presente em cada questão do instrumento de pesquisa utilizado (ZELENY, 1982; ROCHA et al, 2011).

Para a viabilização da análise dos dados levantados, buscou-se verificar a variabilidade nas respostas quanto à concordância e discordância dos atributos (questões). Para isto, matematicamente aplicou-se o seguinte modelo, conforme Zeleny (1982) e Rocha et al. (2011): $\quad d_{i}=\left(d_{i}^{1}, d_{i}^{2}, \ldots, d_{i}^{m}\right)$ os valores normalizados, no qual: $d_{i}^{k}=\frac{x_{i}^{K}}{x_{i}^{*}}$, caracterizando o conjunto D, na forma do i-ésimo atributo. Define-se $D_{i}=\sum_{k=1}^{m} d_{i}^{k} ; \mathrm{i}=1,2, \ldots, \mathrm{n}$. A medida de entropia do contraste de intensidade para o i-ésimo atributo é calculado por e $\left(d_{1}\right)=$ $\alpha=\sum_{k=1}^{m} \frac{d_{i}^{k}}{D_{i}} \operatorname{Ln}\left(\frac{d_{i}^{k}}{D_{1}}\right)$, onde $\alpha=\frac{1}{\varepsilon_{\max }}>0$ e $e_{\max }=\operatorname{Ln}(\mathrm{m})$. Recordando ainda que $0 \leq d_{i}^{k} \leq 1 \mathrm{e}$ 
$d_{i}^{k} \geq 0$. Na ocorrência de todos os $\mathrm{k}$ i d forem idênticos para um dado i, em tal caso $\frac{d_{i}^{k}}{D_{i}}=\frac{1}{n} \mathrm{e}$ $\mathrm{e}\left(d_{i}\right)$ admite valor máximo, ou seja, $e_{\max }=\operatorname{Ln}(\mathrm{m})$. Fixando $\alpha=\frac{1}{\varepsilon_{\max }}$ designa-se $0 \leq$ $\mathrm{e}\left(d_{i}\right) \leq 1$ para todos os $d_{i}{ }^{\prime}$ s. Tal normalização é imprescindível para efeito de comparação. A entropia total de D é determinada por: $E=\sum_{i=1}^{n} e\left(d_{i}\right)$.

Por motivo do peso $\lambda_{i}^{\sim}$ ser opostamente concatenado a e $\left(d_{i}\right)$ é utilizado $1-\mathrm{e}\left(d_{i}\right)$ ao contrário de e $\left(d_{i}\right)$ e normaliza-se para garantir que $0 \leq$ o $\lambda_{i}^{\sim} \leq 1$ e $\sum_{i=1}^{n} \lambda_{i}^{\sim}=1$. Assim, tem-se: $\lambda_{i}^{\sim}=\frac{1}{n-E}\left[1-e\left(d_{i}\right)\right]=\frac{\left[1-e\left(d_{i}\right)\right]}{n-E}$.

\section{APRESENTAÇÃO E ANÁLISE DOS RESULTADOS}

Neste capítulo são apresentados e discutidos os resultados da pesquisa, iniciando com o teste estatístico de confiabilidade (Alfa de Cronbach), em seguida apresentam-se as estatísticas descritivas (média, desvio padrão das dimensões, ranking) e análise multicriterial de cálculo de entropia da informação.

Tabela 1 Estatísticas de confiabilidade

\begin{tabular}{c|c}
\hline Alfa de Cronbach & N de itens \\
\hline 0,866 & 28 \\
\hline
\end{tabular}

Fonte: dados da pesquisa.

Como é possível observar na Tabela 1 , o valor obtido foi de 0,866 . Com este dado é possível inferir que o instrumento (questionário) de pesquisa adaptado de Rodrigues, Esther e Andrade (2017) possui uma ótima fidedignidade para todos os 28 itens do questionário.

Tabela 2 Média e Desvio Padrão das Dimensões

\begin{tabular}{l|c|c}
\hline \multicolumn{1}{c|}{ Dimensão } & Média & Desvio padrão \\
\hline Dim. 1 - Os papeis da Universidade & 5,34 & 1,28167 \\
Dim.2 - Riscos: Universidade e o ambiente externo & 3,99 & 1,92183 \\
Dim.3 - Aprox. Universidade com Empresa e Governo: Motivações & 6,05 & 1,34100 \\
Dim. 4 - Parcerias: a Perspectiva da Dependência De Recursos & 5,44 & 1,57109 \\
\hline
\end{tabular}

Fonte: dados da pesquisa.

Verifica-se na Tabela 2 que na percepção dos atores universitários pesquisados é papel da universidade oferecer ensino, pesquisa e extensão, produzir e difundir conhecimento (Dim. 


\section{REDES INTERINSTITUCIONAIS NA PERSPECTIVA DA HÉLICE TRÍPLICE: O CASO DE UMA \\ UNIVERSIDADE PÚBLICA DO SUL DO BRASIL \\ DOI: https://doi.org/10.5007/1983-4535.2021.e74645}

1). Além disto, não há uma clareza entre os respondentes se a relação da universidade com o ambiente externo pode promover riscos para a universidade (Dim. 2).

A dimensão que obteve maior pontuação foram os motivos que levam a universidade a se aproximar de empresas e governos: obter recursos financeiros, contribuir para o desenvolvimento econômico e social e proporcionar maior inserção dos alunos no mercado. (Dim. 3). A Dimensão 4 obteve a segunda maior pontuação da escala, e refere-se o propósito da universidade buscar parcerias, sob a perspectiva da dependência de recursos.

Com relação a motivação da universidade em se aproximar dos demais membros da hélice tríplice (empresa e governo), formando uma rede de cooperação, segundo Porto et al. (2011) é a viabilização de projetos, estes, por sua vez, contribuem na aproximação e atualização contínua dos acadêmicos com o mercado. Nesta relação, todos os envolvidos da hélice tríplice possuem ganhos. "As motivações que levam à cooperação são distintas para as universidades e as empresas, bem como suas características e necessidades, a intersecção destas encontra-se na necessidade de inovar.” (p. 53)

Tabela 3 Ranking das questões com a pontuação mais alta, por dimensão.

\begin{tabular}{c|c|c|c}
\hline Dimensões & Questões & Likert & Frequência \\
\hline D1 & Q1 & 7 & $55,8 \%$ \\
D3 & Q13 & 7 & $53,5 \%$ \\
D3 & Q14 & 7 & $51,2 \%$ \\
\hline D3 & Q17 & 7 & $55,8 \%$ \\
D4 & Q18 & 7 & $55,8 \%$ \\
D4 & Q19 & 7 & $62,8 \%$ \\
D4 & Q20 & 7 & $65,1 \%$ \\
\hline
\end{tabular}

Fonte: dados da pesquisa.

$\mathrm{Na}$ Tabela 3 realizou-se um ranqueamento, a partir da contagem de frequência das questões que obtiveram uma pontuação mais alta de cada dimensão (likert 7). É possível verificar que a questão que obteve maior pontuação na escala foi a Q 20, da dimensão 4, que se refere a "Obtenção de recursos para a aquisição de equipamentos, laboratórios, etc.". $65,1 \%$ dos respondentes percebem que por meio da obtenção dos recursos externos, é possível adquirir equipamentos e montar laboratórios na universidade. A este respeito, Etzkowitz (1994) destaca que a hélice tríplice possibilita a obtenção de recursos que são externos à universidade, além do governo. Pois, viabiliza-se parcerias com as empresas privadas como fonte para o financiamento de projetos. 


\section{REDES INTERINSTITUCIONAIS NA PERSPECTIVA DA HÉLICE TRÍPLICE: O CASO DE UMA \\ UNIVERSIDADE PÚBLICA DO SUL DO BRASIL \\ DOI: https://doi.org/10.5007/1983-4535.2021.e74645}

Em seguida, calculou-se a entropia da informação de cada componente ou item (Tabela 4).

Tabela 4 Entropia da Informação

\begin{tabular}{|c|c|c|c|}
\hline \multicolumn{2}{|c|}{ Variáveis } & Entropia e $\left(d_{i}\right)$ & Peso \\
\hline \multirow{6}{*}{ Dimensão 1} & D1-Q1 & 0,025451 & 0,166544 \\
\hline & D1-Q2 & 0,016749 & 0,168031 \\
\hline & D1-Q3 & 0,031641 & 0,165486 \\
\hline & D1-Q4 & 0,023592 & 0,166862 \\
\hline & D1-Q5 & 0,022994 & 0,166964 \\
\hline & D1-Q6 & 0,027969 & 0,166114 \\
\hline \multirow{6}{*}{ Dimesão 2} & D2-Q7 & 0,013648 & 0,168155 \\
\hline & D2-Q8 & 0,021666 & 0,166788 \\
\hline & D2-Q9 & 0,021443 & 0,166827 \\
\hline & D2-Q10 & 0,023975 & 0,166395 \\
\hline & D2-Q11 & 0,02178 & 0,031773 \\
\hline & D2-Q12 & 0,166769 & 0,165065 \\
\hline \multirow{5}{*}{ imensão 3} & D3-Q13 & 0,025947 & 0,199174 \\
\hline & D3-Q14 & 0,020202 & 0,200349 \\
\hline & D3-Q15 & 0,020497 & 0,200289 \\
\hline & D3-Q16 & 0,02003 & 0,200384 \\
\hline & D3-Q17 & 0,022866 & 0,199804 \\
\hline \multirow{11}{*}{ Dimensão 4} & D4-Q18 & 0,0254509 & 0,0907916 \\
\hline & D4-Q19 & 0,0251767 & 0,0908171 \\
\hline & D4-Q20 & 0,0251092 & 0,0908234 \\
\hline & D4-Q21 & 0,0266179 & 0,0906828 \\
\hline & D4-Q22 & 0,0286135 & 0,0904969 \\
\hline & D4-Q23 & 0,0234158 & 0,0909812 \\
\hline & D4-Q24 & 0,0214426 & 0,0911650 \\
\hline & D4-Q25 & 0,0215258 & 0,0911572 \\
\hline & D4-Q26 & 0,0193058 & 0,0913641 \\
\hline & D4-Q27 & 0,0224174 & 0,0910742 \\
\hline & D4-Q28 & 0,0270077 & 0,0906465 \\
\hline
\end{tabular}

Fonte: dados da pesquisa.

Após rodar o modelo matemático, utilizado para calcular a entropia da informação de cada questão, levantada no instrumento aplicado para este estudo (Tabela 4), verificou-se que as informações mais relevantes estão presentes na Dimensão 3 "Aproximação da Universidade com Empresa e Governo: Motivações", de acordo com os respondentes, foram: D3Q14 "Contribuir no desenvolvimento econômico e social." (20,03\%) e D3Q16 "Proporcionar maior inserção dos alunos no mercado." (20,03\%). A questão que houve menos informação $(9,05 \%)$ foi a D4Q22 “Obtenção de recursos para a contratação de profissionais especializados (interno: locação de horas na Universidade)." 


\section{REDES INTERINSTITUCIONAIS NA PERSPECTIVA DA HÉLICE TRÍPLICE: O CASO DE UMA \\ UNIVERSIDADE PÚBLICA DO SUL DO BRASIL \\ DOI: https://doi.org/10.5007/1983-4535.2021.e74645}

Analisando os dados desta dimensão, percebe-se que os respondentes consideram importante a aproximação da universidade com empresa e governo. Entendem que esta relação pode promover uma maior inserção dos alunos no mercado de atuação profisssional. Além disto, contribui para o desenvolvimento econômico e social, por meio da produção de pesquisas que promovem soluções demandadas pelas empresas. Este dado corrobora com o entendimento de Etzkowitz (2003), para o autor a proposta de um arranjo entre universidade, empresa e governo, melhora as condições de inovação em uma sociedade baseada no conhecimento, e a universidade produz novos conhecimentos e tecnologia.

As organizações conferem às universidades uma forma de suplementar o seu empenho tecnológico, como fonte de vantagem competitiva com a redução de custos com pesquisas. As empresas dispõem de recursos internos restritos (profissionais qualificados e recursos financeiros) para a produção de pesquisa. Desta forma as universidades são consideradas um receptáculo de conhecimento. As parcerias constituem uma forma dos envolvidos acessarem os recursos que cada membro (hélice tríplice) dispõem. Correntemente, as empresas veem nas universidades uma oportunidade para a suplementação tecnológica, como fonte de vantagem competitiva, atenuando custos com as pesquisas (PORTO, 2006).

Com relação a primeira dimensão "Os papeis da Universidade", composta pelas questões: Q1, Q2, Q3, Q4, Q5 e Q6 do instrumento de Rodrigues, Esther e Andrade (2017), houve uma homogeneização das respostas. Ou seja, as seis questões presentes nesta dimensão possuem em média 16\% de informação (variações entre: 16,54\% e 16,83\%), não apresentando variabilidade acentuada entre elas. Os itens deste fator tratam de questões relacionadas a tríade de ensino, pesquisa e extensão; inovação e visualização de demandas externas; agente de desenvolvimento econômico e social; produtora e difusora de conhecimento.

Como pode-se observar, os papeis percebidos como sendo da universidade estão relacionadas as atividades inerentes a sua concepção. Segundo Meyer Jr. (2014) as universidades possuem um relevante papel na sociedade. Para Porto (2006) as universidades são tidas como um reservatório de conhecimento. De acordo com Melo (2002); Rodrigues; Esther e Andrade (2017) o papel da universidade como membro da hélice tríplice é importante, devido a sua contribuição na produção de conhecimento para potencializar a inovação.

Quanto a dimensão dois, trata dos possíveis riscos da interação da Universidade com o ambiente externo: D2Q7 "Não há riscos para universidade" (16,81\%). As questões D2Q8 até 


\section{REDES INTERINSTITUCIONAIS NA PERSPECTIVA DA HÉLICE TRÍPLICE: O CASO DE UMA \\ UNIVERSIDADE PÚBLICA DO SUL DO BRASIL \\ DOI: https://doi.org/10.5007/1983-4535.2021.e74645}

D2Q11 apresentaram poucas variações (percentuais de explicações entre 16,63\% até 16,68\%) se referem a universidade desviar o foco de suas finalidades públicas para atender demandas privadas; os riscos dependem da forma como essa relação é regulamentada; o risco da universidade entrar com o ônus e as empresas ficarem com o bônus; priorizar somente a ligação com algum setor da sociedade, como as empresas. A questão D2Q12 "Benefícios somente para áreas com maior ligação e coerência com as demandas de mercado" apresenta um percentual ainda menor, de 16,50\%. Assim como foi possível observar na Tabela 2, é interessante ressaltar que nesta dimensão, se evidencia a ausência de clareza dos respondentes quanto os possíveis riscos que a universidade pode estar passível na interação com o ambiente externo.

Por fim, a dimensão quatro "Motivos que levariam a universidade a buscar parcerias com o governo e as empresas, sob a perspectiva da dependência de recursos" apesar de obter a segunda maior pontuação na escala (Tabela 2), apresentou menos informação, conforme a Tabela 4. Infere-se que tal dado se deve ao fato de ter havido menos variabilidade nas respostas, e ter concentrado um percentual maior no valor 7 da escala likert, apresentando uma maior concordância com as assertivas apresentadas.

\section{CONCLUSÃO}

Neste estudo, o objetivo principal foi analisar a percepção dos atores universitários acerca das parcerias interinstitucionais, na perspectiva de uma universidade pública municipal, como membro da hélice tríplice. Os resultados demonstram que na percepção dos atores universitários é papel da universidade oferecer ensino, pesquisa e extensão, produzir e difundir conhecimento (Dim. 1). Verificou-se também que, não há uma clareza entre os respondentes se a relação entre a universidade com o ambiente externo pode trazer riscos para a universidade (Dim. 2). Este dado difere do resultado encontrado na pesquisa de Rodrigues, Esther e Andrade (2017), em que a maioria não visualiza riscos para a universidade, na interação com o ambiente externo.

Duas dimensões obtiveram as maiores pontuações da escala, que foram "os motivos que leva a universidade a se aproximar de empresas e governos" (Dim. 3), e "o propósito da universidade buscar parcerias, sob a perspectiva da dependência de recursos" (Dim. 4). Com relação a motivação da universidade em se aproximar dos demais membros da hélice tríplice, ocorre pela obtenção de recursos financeiros, e pela contribuição que esta relação reflete no 


\section{REDES INTERINSTITUCIONAIS NA PERSPECTIVA DA HÉLICE TRÍPLICE: O CASO DE UMA \\ UNIVERSIDADE PÚBLICA DO SUL DO BRASIL \\ DOI: https://doi.org/10.5007/1983-4535.2021.e74645}

desenvolvimento econômico e social, além de proporcionar uma maior inserção dos futuros profissionais no mercado. Este dado confirma o entendimento de Etzkowitz (2003), segundo o autor a proposta de uma rede de cooperação entre universidade, empresa e governo, melhora as condições de inovação em uma sociedade baseada no conhecimento, e a universidade produz novos conhecimentos e tecnologia.

A dimensão que obteve a segunda maior pontuação na escala, mas apresentou a menor variabilidade entre os respondentes, apresentando uma maior concordância com as assertivas apresentadas, foi a dimensão 4. Esta trata dos motivos que levam a universidade a buscar parcerias com o governo e as empresas, sob a perspectiva da dependência de recursos. Esta dimensão apresentou menos informação, de acordo com a análise multicriterial.

Por fim, espera-se que o presente estudo encoraje futuras pesquisas, como a aplicação do instrumento sobre as parcerias interinstitucionais na perspectiva da hélice tríplice em outras universidades. Assim, sugere-se ampliar a amostra, a fim de verificar a percepção dos atores universitários acerca desta temática, no sentido de verificar possíveis extrapolações em um outro contexto. A replicação desta pesquisa corrobora também para o desenvolvimento da área de estudo, tendo em vista a escassez de trabalhos empíricos sobre as parcerias interinstitucionais e da dependência de recursos como membro da hélice tríplice. Pesquisas nessa área podem ajudar a fomentar o desenvolvimento social, econômico local e do país.

\section{REFERÊNCIAS}

ALMEIDA, Adiel Teixeira de. Processo de decisão nas organizações: construindo modelos de decisão multicritério. São Paulo: Atlas, 2013.

BALESTRIN, Alsones; VERSCHOORE, Jorge Renato; REYES JUNIOR, Edgar. O campo de estudo sobre redes de cooperação interorganizacional no Brasil. RAC-Revista de Administração Contemporânea, v. 14, n. 3, 2010.

BARNEY, Jay. Firm resources and sustained competitive advantage. Journal of management, v. 17, n. 1, p. 99-120, 1991.

BARNEY, Jay B. Is the resource-based "view" a useful perspective for strategic management research? Yes. Academy of management review, v. 26, n. 1, p. 41-56, 2001.

BOURDIEU, Pierre. Escritos de educação. In: Escritos de educação. 2004.

BUSH, Tony. Theories of Educational Management. International Journal of Educational Leadership Preparation, v. 1, n. 2, p. n2, 2006. 
CLEGG, Stewart et al. Handbook de estudos organizacionais. São Paulo: Atlas, 1999.

COLLIS, Jill; HUSSEY, Roger. Business research: A practical guide for undergraduate and postgraduate students. Palgrave macmillan, 2013.

COLOMBO, S. S. Gestão universitária: os caminhos para a excelência. Porto Alegre: Penso, 2013.

CORRAR, Luiz J.; PAULO, Edilson; DIAS FILHO, José Maria. Análise multivariada: para os cursos de administração, ciências contábeis e economia. São Paulo: Atlas, p. 280-323, 2007.

ETZKOWITZ, H. Academic-industry relations: a sociological paradigm for economic development. In: LEYDERSDORFF, L.; VAN DEN BESSLAAR, P. In: Evolutionary economics and chaos theory: new directions in technology studies. London: Printer Publishers, 1994.

ETZKOWITZ, Henry. Innovation in innovation: The hélice tríplice of university-industrygovernment relations. Social science information, v. 42, n. 3, p. 293-337, 2003.

ETZKOWITZ, H.; LEYDESDORFF, L. The dynamics of innovation: from national systems and "mode 2" to hélice tríplice of university-industry-government relations. Research Policy, Amsterdam, v. 29, n. 2, p. 109-123, 2000.

ETZKOWITZ, HENRY; ZHOU, CHUNYAN. Hélice Tríplice: inovação e empreendedorismo universidade-indústria-governo. Estudos Avançados, v. 31, n. 90, p. 23-48, 2017.

GOMES, Luiz Flavio Autran Monteiro; ARAYA, Marcela Cecilia Gonzalez; CARIGNANO, Claudia. Tomada de decisões em cenários complexos: introdução aos métodos discretos do apoio multicritério a decisão. São Paulo: Thomson, 2004.

HAIR JR, J. F. et al. Multivariate Data Analysis: a global perspective. 7th edition. Pearson: 2009.

LYNCH, Richard; BAINES, Paul. Strategy development in UK higher education: towards resource-based competitive advantages. Journal of Higher Education Policy and Management, v. 26, n. 2, p. 171-187, 2004.

MATZENAUER, H.B. Avaliação da sustentabilidade ambiental de municípios Paraibanos: Uma aplicação utilizando o método PROMETHEE II. Tese de Doutorado Apresentada ao Programa de Pós-Graduação em Recursos Hídricos e Saneamento Ambiental da Universidade Federal do Rio Grande do Sul, v. 1, 2003.

MELO, Pedro Antônio de. A cooperação universidade/empresa nas universidades públicas brasileiras. Florianópolis: Universidade Federal de Santa Catarina, 2002, 331f. Tese (Doutorado em Engenharia de Produção) - Programa de Pós-Graduação em Engenharia de Produção, Universidade Federal de Santa Catarina, 2002.

PENROSE, E. The theory of growth of the firm. London: Basil Blackwell, 1959. 
PFEFFER, J.; SALANCIK, G. R. The external control of organizations: a resource dependence perspective. New York: Harper e Row, 1978.

PORTO, Geciane Silveira. A decisão de cooperação universidade-empresa sob a ótica dos líderes de grupos de pesquisa da USP cadastrados no diretório de pesquisa do CNPq. Trabalho apresentado para obtenção do título de Livre Docência, 2006.

PORTO, Geciane Silveira et al. Rede de interações universidade-empresa no Brasil: uma análise de redes sociais. Revista de Economia, v. 37, n. 4, 2011.

PRAHALAD, Coimbatore K.; BETTIS, Richard A. The dominant logic: A new linkage between diversity and performance. Strategic management journal, v. 7, n. 6, p. 485-501, 1986.

PRIEM, Richard L.; BUTLER, John E. Is the resource-based "view" a useful perspective for strategic management research?. Academy of management review, v. 26, n. 1, p. 22-40, 2001.

ROCHA, Irani et al. A presença da entropia da informação no controle orçamentário em ambiente inovador. RAI Revista de Administração e Inovação, v. 8, n. 2, p. 82-105, 2011.

RODRIGUES, I. S.; ÉSTHER, A. B.; ANDRADE, E. P. A relação Indústria-GovernoUniversidade: o caso de uma universidade federal de Minas Gerais. In: EnAnpad, 2017, São Paulo. Trabalhos Apresentados, 2017.

STEWART, Theodor J. Multicriteria Decision Analysis. In: International Encyclopedia of Statistical Science. Springer Berlin Heidelberg, 2011.

TEECE, David J. Towards an economic theory of the multiproduct firm. Journal of Economic Behavior \& Organization, v. 3, n. 1, p. 39-63, 1982.

ULRICH, David; BARNEY, Jay B. Perspectives in organizations: resource dependence, efficiency, and population. Academy of Management Review, v. 9, n. 3, p. 471-481, 1984.

ZELENY, M. Multiple Criteria Decision Making. McGraw-Hill, New York, 1982.

ZOU, Zhi-Hong; YI, Yun; SUN, Jing-Nan. Entropy method for determination of weight of evaluating indicators in fuzzy synthetic evaluation for water quality assessment. Journal of Environmental Sciences, v. 18, n. 5, p. 1020-1023, 2006.

WERNERFELT, Birger. A resource-based view of the firm. Strategic management journal, v. 5, n. 2, p. 171-180, 1984. 